\title{
HERWIRI2.1: Electroweak Corrections for Hadron Scattering
}

\author{
Scott Yost ${ }^{* \dagger}$ \\ The Citadel \\ Charleston, SC 29409, USA \\ and \\ Institute for Nuclear Physics, Polish Academy of Science (IFJ-PAN) \\ Kraków, Poland \\ E-mail: scott.yost@citadel.edu

\section{B.F.L. Ward} \\ Baylor University \\ Waco, TX 76798, USA \\ E-mail: bfl_ward@baylor.edu
}

\begin{abstract}
We describe the program HERWIRI2.1, which implements $\mathscr{O}\left(\alpha^{2}\right)$ photonic radiative corrections exponentiated at the amplitude level (initial state, final state, and initial-final interference) and electroweak corrections to the matrix element by generating events using the KK Monte Carlo to generate the hard process, with quark initial states generated according to PDFs via an LHAPDF interface. The events can be showered internally using HERWIG6.5 or exported and showered using any desired external showering program. Some early results are shown, including comparisons to HERWIG6.5 and HORACE 3.1.
\end{abstract}

Loops and Legs in Quantum Field Theory

24-29 April 2016

Leipzig, Germany

\footnotetext{
*Speaker.

${ }^{\dagger}$ This work and its presentation were supported in part by grants from The Citadel Foundation.
} 


\section{Introduction}

Vector boson production plays an important role at the LHC, both as a benchmark process for precision tests of the Standard Model and as a background in searches for new physics.[1] It is desired to measure this process at the $1 \%$ level, but electroweak corrections alone are typically several percent, as seen in the studies of Ref. [2, 3]. A number of programs are presently available for calculating electroweak corrections to hadron scattering, and a comprehensive review of the state of the art in calculating vector boson observables at the LHC can be found in Ref. [1], which presents tuned comparisons using HORACE [4, 5], PHOTOS [6, 7, 8], SANC [9], WINHAC [10, 11, 12], WZGRAD [13, 14, 15], and FEWZ3 [16], together with programs implementing higher order QCD effects, which are not presently included in HERWIRI2.1, but will be implemented in a future version.

The name HERWIRI is an acronym for High Energy Radiation With Infra-Red Improvements, and recognizes the role HERWIG [17, 18, 19] has in the project. HERWIRI2.x, which concentrates on electroweak and exponentiated photonic corrections, is independent of HERWIRI1.x [20, 21], another project under the HERWIRI umbrella which implements a non-abelian analog of the the amplitude-level YFS-inspired [22] exponentiation developed for KKMC [23] to modify the soft behavior of the parton shower.

HERWIRI2.1 adapts KKMC, which was developed as a high-precision generator for $e^{+} e^{-} \rightarrow$ $\gamma, Z \rightarrow f \bar{f}$ for LEP physics, to hadronic collisions. An early, unreleased version of HERWIRI2 [24] was based on the original KKMC and a reweighting scheme. However, KKMC was later upgraded to version 4.22 [27], which supports quark initial states, and this version became the basis of HERWIRI2.1, which generates quark initial states using an LHAPDF [25] interface integrated into KKMC 4.22.

HERWIRI2 generates a hard process for $p p$ or $p \bar{p}$ scattering including ISR and FSR photons added via KKMC, and the events may be exported for external showering, or showered internally using HERWIG6.5, which is included in any case as a means of incorporating HEPEVT bookkeeping and PDF management in a form which is consistent with HERWIG. Tau lepton events may be passed to a shower as-is, or tau decay may be implemented via TAUOLA $[28,29,30]$ and PHOTOS, which are incorporated into KKMC.

A HERWIRI2.1 run begins with an initialization phase when parameters are set, both in KKMC and HERWIG6.5. This insures compatible parameters if the internal HERWIG shower is used. Even without an internal shower, HERWIG routines are used for HEPEVT and PDF management. In addition, the underlying MC generator, FOAM, creates an adaptive grid for the subsequent event generation and calculates a crude integral, as explained in Section 3.

\section{EEX and CEEX Exclusive Exponentiated Cross Sections}

HERWIRI2.1 incorporates KKMC's options for calculating exponentiated ISR and FSR using either cross-section level exponentiation (EEX) or amplitude-level exclusive exponentiation (CEEX). The default CEEX mode includes initial-final interference (IFI) effects and exact emission to order $\alpha, \alpha^{2} L^{2}$, and $\alpha^{2} L$, where $L=\ln \left(p^{2} / m^{2}\right)$ with the hard process scale $p^{2}$ and the relevant mass $m$, which is the final fermion mass for FSR and quark current mass for ISR. This is referred to 
as $\mathscr{O}\left(\alpha^{2}\right)$-pragmatic.[23] EEX mode includes exact order $\alpha$, as well as exact order $\alpha^{2} L^{2}, \alpha^{2} L$, and $\alpha^{3} L^{3}$, which is referred to as $\mathscr{O}\left(\alpha^{3}\right)$-pragmatic. The most precise $\mathscr{O}\left(\alpha^{2}\right)$ corrections in KKMC may be found in Ref. [31]. Electroweak boson mixing enters at the $\alpha^{2}$ non-logarithmic order, so EEX includes most-precise $\mathscr{O}\left(\alpha^{2}\right)$ corrections for which full electroweak gauge invariance can be maintained in QED alone. When run in CEEX mode, alternative weights are provided for EEX and lower orders in $\alpha$ as well. There is also a CEEX weight without IFI, for comparison.

The cross section takes the form

$$
\sigma=\sum_{i} \int d x_{1} d x_{2} d v f_{i}\left(p^{2}, x_{1}\right) f_{\bar{i}}\left(p^{2}, x_{2}\right) \sigma\left(p^{2}, v\right) \delta\left(p^{2}-s x_{1} x_{2}\right)
$$

where $p^{2}=\left(p_{1}+p_{2}\right)^{2}$ for initial quark momenta $p_{1}$ and $p_{2}$ given as fractions $p_{j}=x_{j} P_{j}$ of the proton or anti-proton beam momenta $P_{j}(j=1,2), i$ indexes all relevant quark and antiquark flavors, and $f_{i}$ are the parton distribution functions. In the EEX case, the squared invariant mass after ISR is $\left(p-K_{I}\right)^{2}=p^{2}(1-v)$, where $K_{I}$ is the total initial photon momentum, and

$$
\begin{aligned}
\sigma\left(p^{2}, v\right)= & \sum_{n=0}^{\infty} \sum_{n^{\prime}=0}^{\infty} \frac{1}{n ! n^{\prime} !} \exp \left(Y_{I}\left(\Omega_{I} ; p^{2}\right)\right) \int D q_{1} D q_{2} \exp \left(Y_{F}\left(\Omega_{F} ; q^{2}\right)\right) \\
& \int \prod_{j=1}^{n} D k_{j}\left(1-\Theta\left(\Omega_{I} ; k_{j}\right)\right) \int \prod_{j^{\prime}=1}^{n^{\prime}} D k_{j^{\prime}}^{\prime}\left(1-\Theta\left(\Omega_{F} ; k_{j^{\prime}}\right)\right) \delta\left(K_{I}-\sum_{j=1}^{n} k_{j}\right) \\
& \delta\left(v-\frac{\left(2 p-K_{I}\right) \cdot K_{I}}{p^{2}}\right) \rho_{\mathrm{EEX}}^{\left(n, n^{\prime}\right)}\left(p_{1}, p_{2} ; q_{1}, q_{2} ; k_{1}, \ldots, k_{n} ; k_{1}^{\prime}, \ldots, k_{n^{\prime}}^{\prime}\right)
\end{aligned}
$$

Here, $D k$ represents the Lorentz invariant phase space density for momentum $k, \Omega_{I}$ and $\Omega_{F}$ are soft photon regions for ISR and FSR, $\Theta(\Omega, k)=1$ or 0 depending on whether $k \in \Omega$ or not, $\widetilde{S}_{I, F}$ are initial and final state soft photon factors, and $Y_{I, F}$ are initial and final state YFS factors. The density $\rho_{\mathrm{EEX}}^{n, n^{\prime}}$ with $n$ ISR and $n^{\prime}$ FSR photons is constructed from a combination of soft photon factors $\widetilde{S}(k)$ and $N$-photon YFS residuals $\bar{\beta}_{N}\left(k_{1}, \ldots k_{N}\right)$,

$$
\rho_{\mathrm{EEX}}^{\left(n, n^{\prime}\right)}=\prod_{j=1}^{n} \widetilde{S}_{j} \prod_{j^{\prime}=1}^{n^{\prime}} \widetilde{S}_{j^{\prime}}\left\{\bar{\beta}_{0}+\sum_{j=1}^{n} \frac{\bar{\beta}_{1}\left(k_{j}\right)}{\widetilde{S}_{I}\left(k_{j}\right)}+\sum_{j=1}^{n^{\prime}} \frac{\bar{\beta}_{1}\left(k_{j^{\prime}}\right)}{\widetilde{S}_{F}\left(k_{j}\right)}+\ldots\right\} .
$$

Detailed expressions may be found in Ref. [23], with the incoming electron replaced by a quark. The YFS residuals are all evaluated at a scale $s_{X} \equiv p^{2}(1-v)$, which represents the scale of the hard $\gamma / Z$ process.

In the CEEX case, the photons cannot be sorted unambiguously into ISR and FSR subsets, so the sum over all such possibilities is performed, and $v$ plays an analogous role for the sum over ISR photons in a given partition. The analog of (2.2) is

$$
\begin{aligned}
& \sigma_{\mathrm{CEEX}}\left(p^{2}, v\right)=\sum_{N=0}^{\infty} \sum_{\mathscr{P}} \frac{1}{N !} \int D q_{1} D q_{2} \exp \left(Y_{F}\left(\Omega ; p_{1}, p_{2}, q_{1}, q_{2}\right)\right) \int \prod_{j=1}^{N} D k_{j}\left(1-\Theta\left(\Omega ; k_{j}\right)\right) \\
& \delta\left(v-\frac{\left(2 p-K_{I}(\mathscr{P})\right) \cdot K_{I}(\mathscr{P})}{p^{2}}\right) \delta\left(K_{I}(\mathscr{P})-\sum_{j=1}^{n(\mathscr{P})} k_{j}\right) \rho_{\mathrm{CEEX}}^{(N, \mathscr{P})}\left(p_{1}, p_{2} ; q_{1}, q_{2} ; k_{1}, \ldots, k_{N}\right),
\end{aligned}
$$

where $\mathscr{P}$ is a partition of $N$ photons into ISR and FSR subsets, which are summed in all possible ways with $n(\mathscr{P})$ ISR photons and $n^{\prime}(\mathscr{P})=N-n(\mathscr{P})$ FSR photons, and $\rho_{\mathrm{CEEX}}^{(N, \mathscr{P})}$ is constructed as 
a sum of absolute squares of helicity amplitudes

$$
\mathscr{M}_{N}^{\mathscr{P}}=\prod_{i=1}^{n(\mathscr{P})} \mathscr{S}_{I}\left(k_{i}\right) \prod_{i^{\prime}=1}^{n^{\prime}(\mathscr{P})} \mathscr{S}_{F}\left(k_{i^{\prime}}\right)\left\{\widehat{\beta}_{0}+\sum_{j=1}^{n} \frac{\widehat{\beta}_{1}\left(k_{j}\right)}{\mathscr{S}_{I}\left(k_{j}\right)}+\sum_{j^{\prime}=1}^{n^{\prime}} \frac{\widehat{\beta}_{1}\left(k_{j^{\prime}}\right)}{\mathscr{S}_{I}\left(k_{j^{\prime}}\right)}+\ldots\right\},
$$

where the $\widehat{\beta}_{N}$ are spinor-amplitude residuals and the absolute squares of the soft-photon spinors $\mathscr{S}_{I}$ and $\mathscr{S}_{F}$ yield $\widetilde{S}_{I}$ and $\widetilde{S}_{F}$, respectively, while the cross terms are responsible for IFI corrections. A common soft-photon set $\Omega$ is used for both ISR and FSR, since the assignment to these sets is arbitrary. Again, the detailed forms may be found in Ref. [23], with incoming quarks replacing electrons.

\section{Primary Distribution}

The cross section is constructed as a Monte Carlo integral over $p \equiv \sqrt{p^{2}}, x_{1}, v$ and a sum over quark and antiquark flavors. The underlying FOAM MC generates these according to a crude primary distribution which it integrates during the event generation. The $\mathrm{C}++$ version of FOAM is used, which allows the quark flavor dimension to be treated separately, with a predefined separation into five flavors and five anti-flavors, and no cell division. The remaining three dimensions are divided into simplicial cells during the exploration phase.

Specifically, the default mappings used are

$$
p^{-2}=\frac{1-r_{1}}{p_{\min }^{2}}+\frac{r_{1}}{p_{\max }^{2}}, \quad x_{1}=\left(\frac{p^{2}}{s}\right)^{r_{2}}, \quad v=v_{\max } r_{3}^{1 / \gamma},
$$

for $0 \leq r_{i}<1$, where $\gamma=\bar{\gamma}-2 Q_{i}^{2} \alpha / \pi$ and

$$
\bar{\gamma}=\frac{2 Q_{i}^{2} \alpha}{\pi} \ln \left(\frac{(1+\beta)^{2}}{m_{i}^{2}}\right)
$$

for a quark with charge $Q_{i}$ and current mass $m_{i}$, with $\beta=\sqrt{1-4 m_{i}^{2} / s}$.

The FOAM integrand for the crude MC integral is

$$
\begin{aligned}
\rho_{i}\left(p^{2}, v\right)= & 2 N_{q} p^{4}\left(p_{\max }^{-2}-p_{\min }^{-2}\right) \ln \left(\frac{p^{2}}{s}\right) \frac{\bar{\gamma}}{2 \gamma}\left(1+(1-v)^{-1 / 2}\right) v_{\max }^{\gamma}\left(\frac{v}{v_{\min }}\right)^{2 Q_{i}^{2} \alpha / \pi} \\
& f_{i}\left(p^{2}, x_{1}\right) f_{\bar{i}}\left(p^{2}, x_{2}\right) \sigma_{\mathrm{Born}}\left(p^{2}(1-v)\right),
\end{aligned}
$$

with quark or antiquark label $i$ chosen by dividing the unit interval into $2 N_{q}$ equal intervals and selecting $i$ according to the box into which the first random variable falls.

\section{Electroweak Matrix Element Corrections}

Electroweak matrix element corrections are added using version 6.21 of the DIZET [32] package developed for ZFITTER [33]. The $\gamma$ and $Z$ propagators are multiplied by vacuum polarization factors of the form

$$
H_{\gamma}=\frac{1}{2-\Pi_{\gamma}}, \quad H_{Z}=4 \sin ^{2}\left(2 \theta_{\mathrm{W}}\right) \frac{\rho_{\mathrm{EW}} G_{\mu} M_{Z}^{2}}{8 \pi \alpha \sqrt{2}}
$$


Vertex corrections are incorporated into the coupling of $Z$ to $f$ via form factors in the vector coupling:

$$
g_{V}^{(Z, f)}=\frac{T_{3}^{(f)}}{\sin \left(2 \theta_{W}\right)}-Q_{f} F_{\mathrm{V}}^{(f)}(s) \tan \theta_{W} .
$$

Box diagrams contain these plus a new angle-dependent form-factor in the doubly-vector component:

$$
g_{V}^{(Z, i)} g_{V}^{(Z, f)}=\frac{1}{\sin ^{2}\left(2 \theta_{W}\right)}\left(T_{3}^{(i)} T_{3}^{(f)}-2 T_{3}^{(i)} Q_{f} F_{\mathrm{v}}^{(f)}(s)-2 Q_{i} T_{3}^{(f)} F_{\mathrm{v}}^{(i)}(s)+4 Q_{i} Q_{f} F_{\mathrm{box}}^{(i, f)}(s, t)\right) .
$$

The quantities in these expressions are tabulated at the beginning of the run for hard process energies up to $1040 \mathrm{GeV}$, the highest energy supported by the KKMC interface to DIZET. When EW corrections are required for higher energies, they are calculated as needed.

\section{Results and Comparisons}

In this section, we present results both with and without a hadron shower for proton collisions with a $\mathrm{CM}$ energy of $8 \mathrm{TeV}$, using a cut $50 \mathrm{GeV}<p<200 \mathrm{GeV}$ on the generated quark scale $p=\sqrt{p^{2}}$. In these tests, only muons are generated. The IR boundary is defined by setting a minimum value $v_{\min }=10^{-3}$ in (2.2) or (2.4). In principle, $v_{\min }$ could be set to $10^{-5}$ or less, but smaller values lead to lower efficiency. Electroweak parameters are taken from the benchmark choices in Ref. [1]. HERWIRI2 was run in variable-width mode for these tests. Comparisons are made to HERWIG6.5 and HORACE3.1, which use fixed widths, so the $Z$ mass and width parameters were redefined in those programs as suggested by Ref. [1] to maximize compatibility. All plots are for 25 million event runs with variable weights.

Figs. 1 - 3 show results for a run showered with HERWIG6.5. The choice of shower was made largely for simplicity, since HERWIG6.5 is already used for some PDF support and bookkeeping functions in HERWIRI2.1, but also because it was the basis for the studies of Refs. [2] and [3]. HERWIRI2.1 results are shown for three cases: the best full CEEX and EEX results with ISR and FSR (red), the CEEX result restricted to FSR only (blue), and a result with no photons (green) generated by HERWIRI2.1 using HERWIG's hard cross section. These are compared to HERWIG's result (black). The cross sections in each case are shown in Table 1. The best EEX result agrees with the CEEX cross section to $0.04 \%$. A comparison is also shown for HORACE3.1, with the same parameters. The HORACE comparison should be considered preliminary until it has been more carefully tuned, but the agreement of the signs and general magnitude appear promising.

Figures 1 - 3 show a collection of results for 25-million event runs of HERWIRI2.1 in CEEX mode ( $\mathscr{O}\left(\alpha^{2}\right)$-pragmatic), both the full ISR+FSR result (which includes IFI as well) in red and FSR alone in blue, showered with HERWIG6.5. The result of HERWIG6.5 alone is shown for comparison. Figures 4 - 6 show results of 25 -million event runs without a shower, in which case comparisons to the HERWIG6.5 hard process (in black) and to HORACE3.1 (in green) are shown. While the cuts are different from the studies in Refs. [2] and [3], the sign and general order of magnitude are as anticipated. 


\begin{tabular}{|l|l|l|c|}
\hline Generator & Photons & Cross Section & \% Dif. \\
\hline HERWIG6.5 & None & $1039.6 \pm 0.2 \mathrm{pb}$ & - \\
HERWIRI2.1 & CEEX ISR+FSR & $986.21 \pm 0.26 \mathrm{pb}$ & $-5.1 \%$ \\
HERWIRI2.1 & EEX ISR+FSR & $985.82 \pm 0.26 \mathrm{pb}$ & $-5.2 \%$ \\
HERWIRI2.1 & CEEX FSR & $986.05 \pm 0.11 \mathrm{pb}$ & $-5.2 \%$ \\
HERWIRI2.1 & None (HERWIG Born) & $1038.69 \pm 0.08 \mathrm{pb}$ & $-0.09 \%$ \\
HORACE3.1 & FSR & $1009.15 \pm 0.79 \mathrm{pb}$ & $-2.9 \%$ \\
\hline
\end{tabular}

Table 1: Cross sections for the showered tests. All results are for 25 million events. Differences are relative to HERWIG6.5.
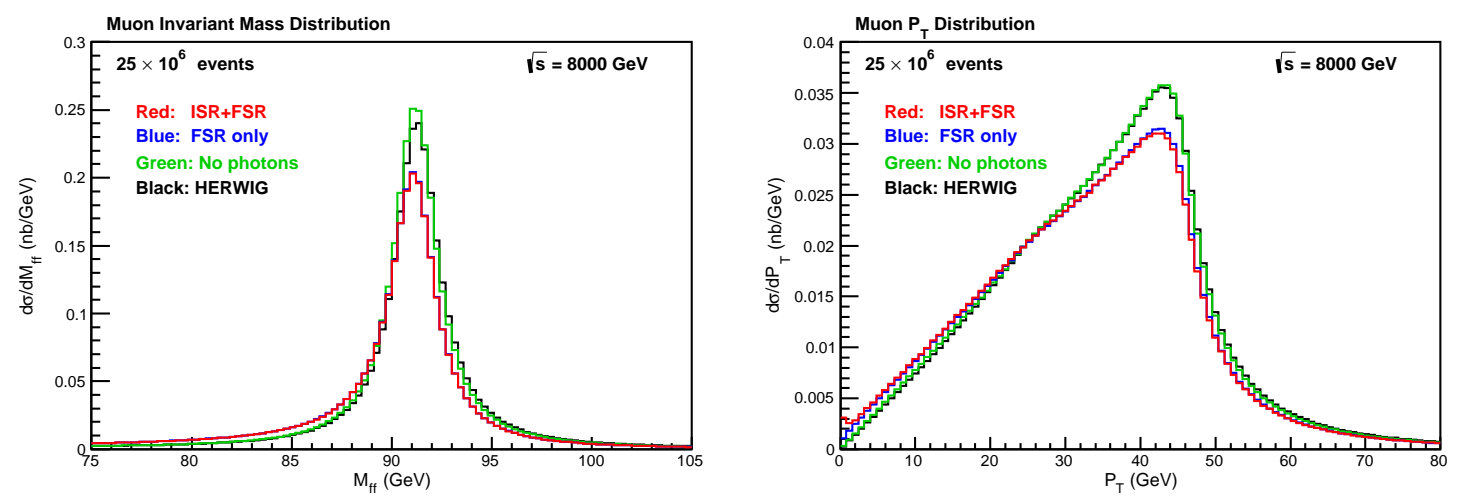

Figure 1: Muon invariant mass and transverse momentum distributions for HERWIRI2.1 for ISR + FSR (red) and for FSR alone (blue), showered by HERWIG6.5. The green distribution is generated by HERWIRI2.1 using HERWIG's hard cross section and the black distribution is made by HERWIG6.5 alone.

\section{Conclusions and Outlook}

We have described the event generator HERWIRI2.1 for $p p$ or $p \bar{p}$ collisions with order $\alpha$ electroweak matrix element corrections and order $\alpha^{2}$-pragmatic CEEX photon emission (ISR, FSR, and IFI), and presented some of its first results, including comparisons to HERWIG and HORACE. The early results are promising and generally in accord with expectations. Further comparisons with more careful tuning and a variety of final fermions and cuts will be forthcoming, as will showering with a variety of modern generators. We also intend to add NLO QCD corrections in a factorized approximation.

\section{Acknowledgments}

We acknowledge the hospitality of the CERN theory division, which contributed greatly to the completion of HERWIRI2.1. S. Yost acknowledges the hospitality and support of the Theoretical Physics Division of the Institute for Nuclear Physics of the Polish Academy of Science and a sabbatical funded by The Citadel Foundation. S. Yost also acknowledges hospitality and support from V. Halyo, D. Marlow, and Princeton University during development of HERWIRI2.0, which also received support from a U.S. Department of Energy grant. 

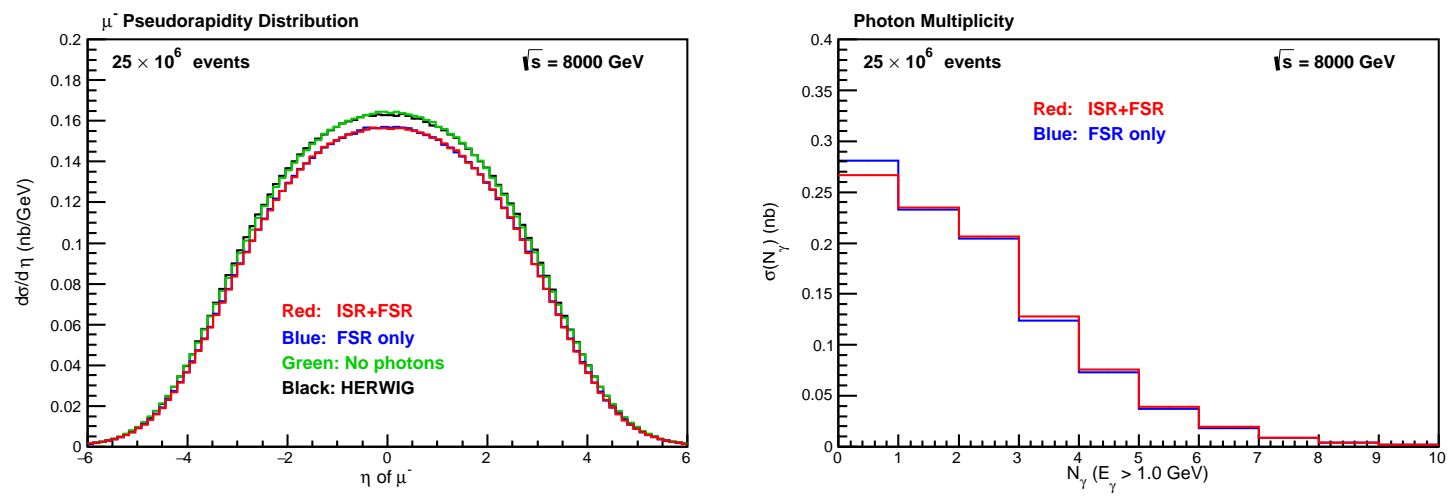

Figure 2: Muon pseudorapidity distribution plotted as in the previous figure, and the number of photons having energy greater than $1 \mathrm{GeV}$ for HERWIRI2.1 with ISR + FSR (red) and FSR alone (blue), showered by HERWIG6.5.
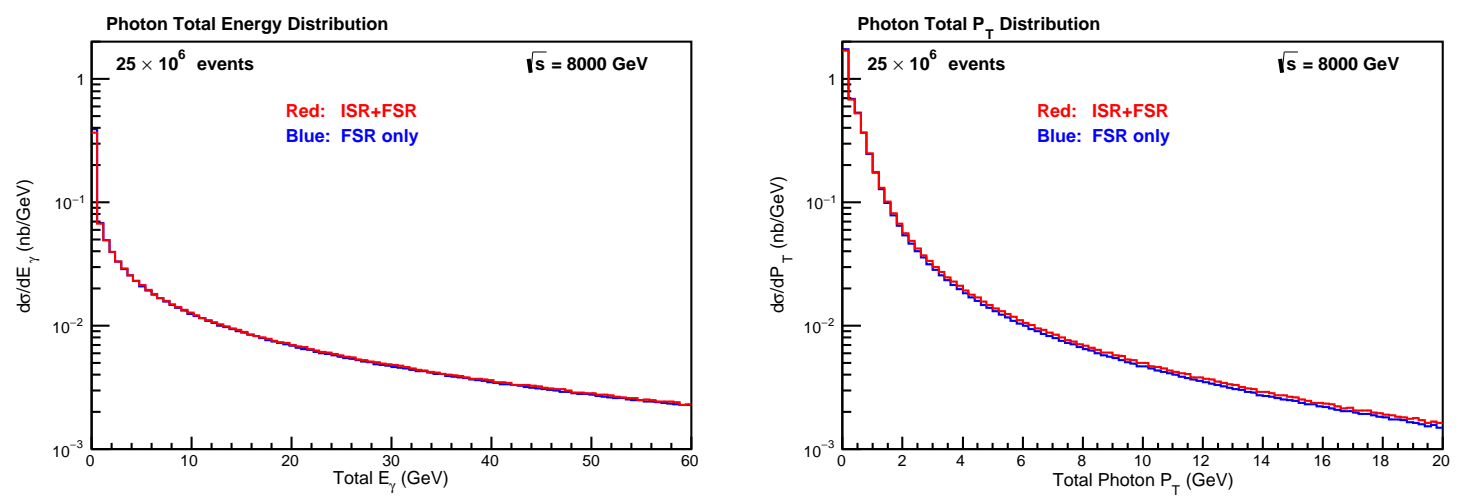

Figure 3: Photon total energy distribution and total photon transverse momentum for HERWIRI2.1 showered by HERWIG6.5, plotted as in the previous figure.

\section{References}

[1] S. Alioli, et al., Precision Studies of Observables in $p p \rightarrow W \rightarrow l v_{l}$ and $p p \rightarrow \gamma, Z \rightarrow l^{+} l^{-}$processes at the LHC, arXiv:1606.02330.

[2] N.E. Adam, V. Halyo, and S.A. Yost, JHEP 05 (2008) 062 (arXiv:0802.3251); ibid., JHEP 11 (2010) 074 (arXiv:1006.3766).

[3] N.E. Adam, V. Halyo, S.A. Yost, and W.-H. Zhu, JHEP 09 (2008) 133 (arXiv:0808.0758).

[4] C.M. Carloni Calame, G. Montagna, O. Nicrosini, and M. Treccani, JHEP 05 (2005) 019 (arXiv:hep-ph/0502218).

[5] C.M. Carloni Calame, G. Montagna, O. Nicrosini, and A. Vicini, JHEP 0612 (2006) 016 (arXiv:hep-ph/0609170); ibid., JHEP 0710 (2007) 109 (arXiv:0710.1722).

[6] E. Barberio, B. van Eijk, and Z. Wass, Comput. Phys. Commun. 66 (1991) 115; E. Barberio and Z. Wa̧s, Comput. Phys. Commun. 79 (1994) 291.

[7] P. Golonka and Z. Wa̧s, Eur. Phys. J. C45 (2006) 97 (arXiv:hep-ph/0506026); ibid., Eur. Phys. J. C50 (2007) 53 (arXiv:hep-ph/0604232). 

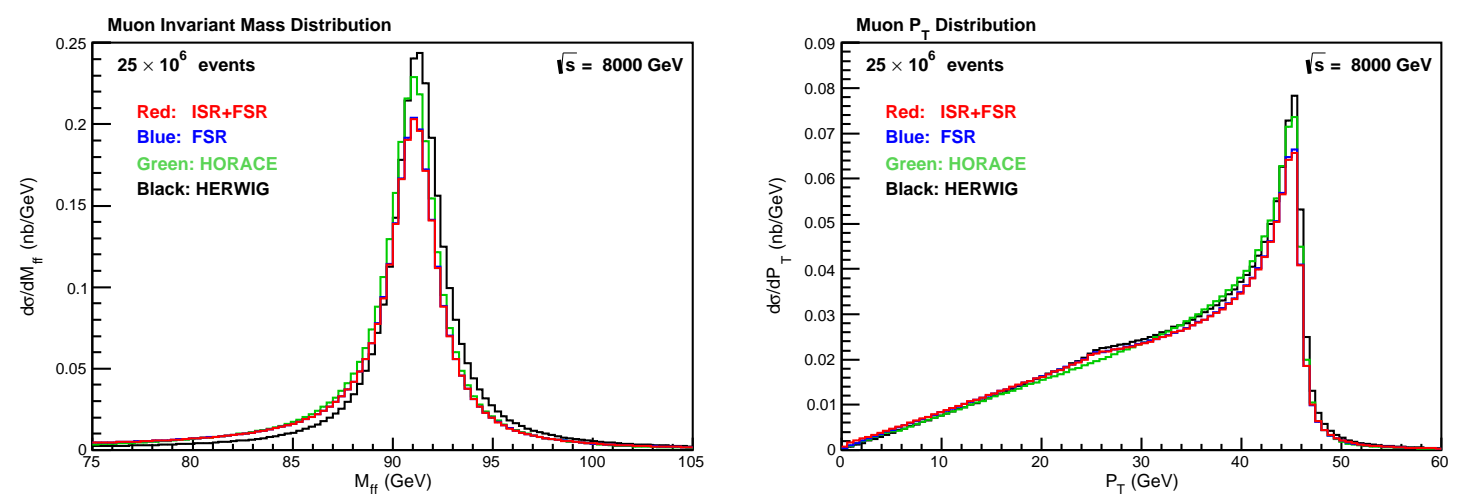

Figure 4: Muon invariant mass and transverse momentum distributions for HERWIRI2.1 with ISR + FSR (red) and FSR only (blue), compared to HORACE3.1 (green) and the unshowered HERWIG6.5 hard cross section (black).
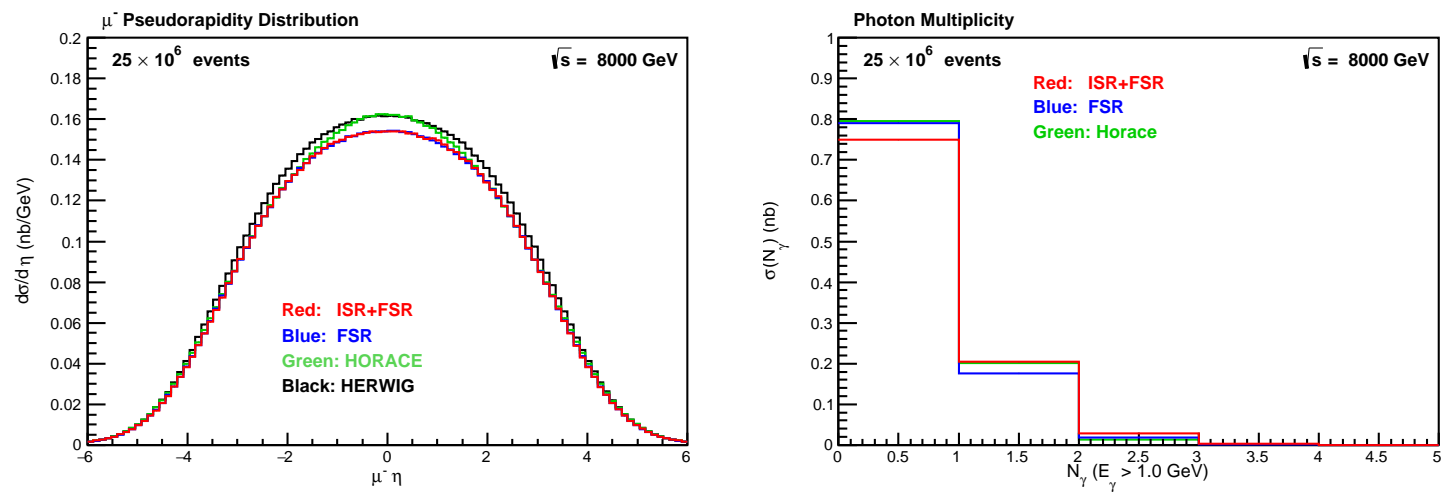

Figure 5: Muon pseudorapidity distribution plotted as in the previous figure and the number of photons with energy greater than $1 \mathrm{GeV}$ for HERWIRI2.1 with ISR + FSR (red) and FSR only (blue), compared to HORACE3.1 (green).

[8] N. Davidson, T. Przedzinski, and Z. Wass, Comput. Phys. Commun. 199 (2016) 86 (arXiv:1011.0937).

[9] A. Arbuzov, et al., Eur. Phys. J. C46 (2006) 407 (arXiv:hep-ph/0506110); ibid., Eur. Phys. J. C54 (2008) 451 (arXiv:0711.0625).

[10] W. Płaczek and S. Jadach, Eur. Phys. J. C29 (2003) 325 (arXiv:hep-ph/0302065).

[11] W. Płaczek, PoS EPS-HEP2009 (2009) 340 (arXiv:0911.0572).

[12] W. Płaczek, S. Jadach, and M.W. Krasny, Acta Phys. Polon. B44 (2013) 2171 (arXiv:1310.5994).

[13] U. Baur, S. Keller, and D. Wackeroth, Phys. Rev. D59 (1999) 013002 (arXiv:hep-ph/9807417).

[14] U. Baur, O. Brein, W. Hollik, C. Schappacher, and D. Wackeroth, Phys. Rev. D65 (2002) 033007 (arXiv:hep-ph/0108274).

[15] U. Baur and D. Wackeroth, Phys. Rev. D70 (2004) 073015 (arXiv:hep-ph/0405191).

[16] Y. Li and F. Petriello, Phys. Rev. D86 (2012) 094034 (arXiv:1208.5967).

[17] G. Marchesini, et al., Comput. Phys. Commun. 67 (1992) 465.

[18] G. Corcella, et al., JHEP 0101 (2001) 010. 

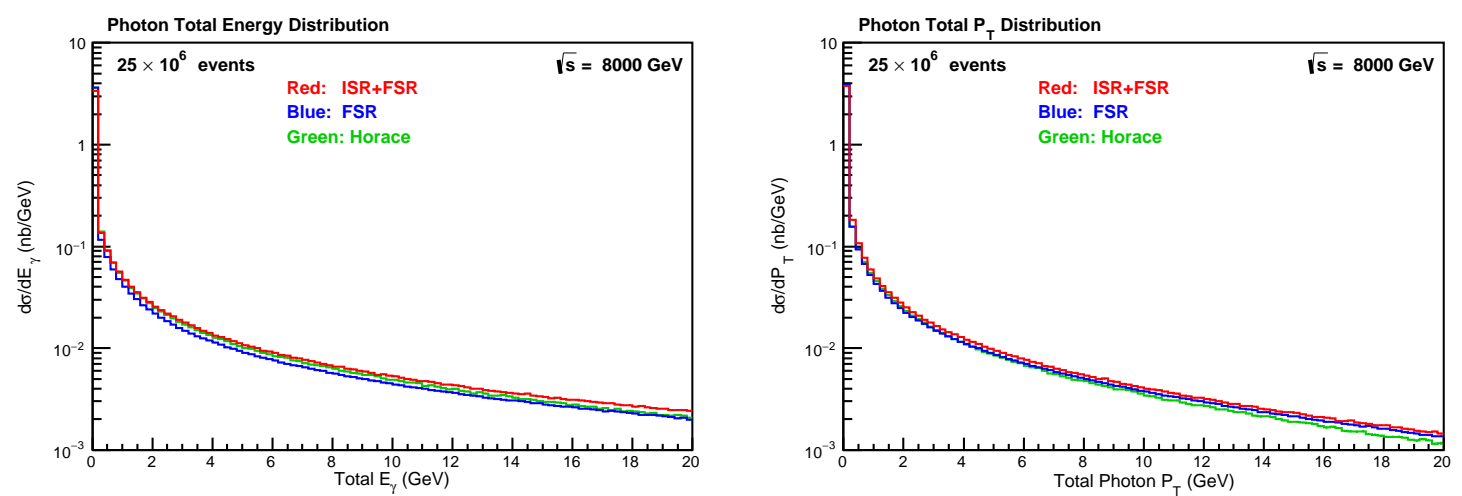

Figure 6: Total photon energy and total photon transverse momentum for HERWIRI2.1 compared to HORACE3.1 as in the previous figure.

[19] S. Gieseke, et al., JHEP 0402 (2004) 005.

[20] S. Joseph, S. Majhi, B.F.L. Ward, and S.A. Yost, Phys. Lett. B685 (2010) 283 (arXiv:0906.0788); ibid., Phys. Rev. D81 (2010) 076008 (arXiv:1001.1434); ibid., Mod. Phys. Lett. A25 (2010) 2207 (arXiv:1001.2730).

[21] S.K. Majhi, A. Mukhopadhyay, B.F.L. Ward, and S.A. Yost, Phys. Lett. B719 (2013) 367 (arXiv:1208.4750); ibid., Ann. Phys. 350 (2014) 485 (arXiv:1305.0023).

[22] D.R. Yennie, S. Frautschi, and H. Suura, Ann. Phys. 13 (1961) 379.

[23] S. Jadach, B.F.L. Ward, and Z. Wass, Phys. Lett. B449 (1999) 97 (arXiv:hep-ph/9905453); ibid., Phys. Rev. D63 (2001) 113009 (arXiv:hep-ph/0006359).

[24] S.A. Yost, V. Halyo, M. Hejna, and B.F.L. Ward, PoS (RADCOR2011) (2012) 017 (arXiv:1201.5906); ibid., PoS (ICHEP2012) (2013) 017 (arXiv:1311.5266).

[25] A. Buckley et al., Eur. Phys. J. C75 (2015) 3, 132 (arXiv:1412.7420).

[26] S. Jadach, Comput. Phys. Commun. 130 (2000) 244 (arXiv:physics/99100004); ibid., Comput. Phys. Commun. 152 (2003) 55 (arXiv:physics/0203033).

[27] S. Jadach, B.F.L. Ward, and Z. Wa̧s, Phys. Rev. D88 (2013) 1114022 (arXiv:1307.4037).

[28] S. Jadach, Z. Wa̧s, and J.H. Kuehn, Comput. Phys. Commun. 64 (1991) 275.

[29] M. Jezabek, Z. Wạs, S. Jadach, and J.H. Kuehn, Comput. Phys. Commun. 70 (1992) 69.

[30] S. Jadach, Z. Wa̧s, R. Decker, and J.H. Kuehn, Comput. Phys. Commun. 76 (1993) 361.

[31] S. Jadach, M. Melles. B.F.L. Ward, and S.A. Yost, Phys. Rev. D65 (2002) 073030 (arXiv:hep-ph/0109279).

[32] A. Akhundov, D. Bardin, M. Bilenky, P. Christova, S. Riemann, T. Riemann, M. Sachwitz, and H. Vogt, DIZET6.21.

[33] A. Akhundov, D. Bardin, and T. Riemann, Phys. Lett. B166 (1986) 111. 